\title{
Automated Meter Reading System - A Study
}

\author{
Satish \\ Palaniappan \\ SSNCE, Anna \\ University \\ OMR, Kalavakkam, \\ Tamilnadu 603110
}

\author{
Raghul Asokan \\ SSNCE, Anna \\ University \\ OMR, Kalavakkam, \\ Tamilnadu 603110
}

\author{
Srinivas \\ Bharathwaj \\ SSNCE, Anna \\ University \\ OMR, Kalavakkam \\ Tamilnadu 603110
}

\author{
Sujaudeen N \\ Assistant Professor, \\ CSE \\ SSNCE, Anna \\ University \\ OMR, Kalavakkam, \\ Tamilnadu 603110
}

\begin{abstract}
Automated Meter reading systems are a invaluable technological advancement that can lead to a better standard of living, owing to the fact that metering has become a part and parcel of our mundane lives. It solves many issues of the traditional meter reading system like need for human resources, efficiency, accuracy, delayed work, unavailability of customer during metering visit by employee, etc. Moreover it is more economical and helps to save energy in a more efficient and effective way. Furthermore it has a very notable advantage of having the ability to predict the energy demands of the future, starting from every household to the entire planet. Automated meter reading systems have been implemented using many different technologies like GSM, ZigBee, PLC, D-SCADA, WiMAX and Hybrid Technologies that comprises of a mixture of the above. This survey paper describes the working models, strengths and weaknesses of each technology by considering various factors like feasibility, cost, reliability, efficiency, maintenance and user experience. This paper not only surveys the existing Automated Metering systems but also provides an abstract view of developing the most optimal automated meter reading system.
\end{abstract}

\section{General Terms}

Automatic Meter Reader, Smart Energy Meter.

\section{Keywords}

AMRS, Smart Meters, Zigbee, GSM, Scada, PLC, WiMax.

\section{INTRODUCTION}

Electrical power has become indispensable to human survival and progress which leads to the enhancement of the people's standard of life by the introduction of automation in to energy distribution and management. With the constant development in technology, the need of automated meter reading systems is also increasing. The technology of e-metering (Electronic Metering) has gone through rapid technological advancements and there is increased demand for a reliable and efficient Automatic Meter Reading (AMR) system.

The traditional meter reading process involves using the analog meters to collect the data of the energy consumed and display it either on a number dial or a digital display. The service provider person comes to the place of the meter and notes down the reading at the end of every billing cycle. But the traditional meter reading process not only wastes labor human power, but also is error prone. The procedures of sending the bills to customer are very laborious and cumbersome. The conventional process is time consuming as well. Another major problem in this system is that the readings cannot be taken if no one is available at the home or where the meter is located. The current system does not provide any scope for the user to conserve energy or does it provide energy consumption predictions for near future that enables the user to act in a more planned way. There are many such problems that cause inconvenience to the power provider as well as the consumers. Even though the conventional meters were replaced with more efficient electronic energy meters these problems still persists.

Automatic meter reading system is a technology which is used to gather data from energy metering devices and transfer it to a central station in order to process it for billing purposes. Automatic meter reading system helps the customer and energy provider to access the accurate and updated data from the meters. AMR system can fetch energy consumption in a hourly, monthly, yearly basis on request or even in Real Time. This Real time energy usage can be seen by the users to control the use of power and be more economical. With the help of the collected data the service provider will be able to send energy saving ideas to the users. This kind of real time data collected from each of the individual houses is really a boon to data scientists, who use machine learning and data mining tools to build a predictive model over this valuable data to predict the future energy demands starting from every single house, area, city to the entire planet. Thus leading to sophisticated and predictive energy production, conservation and management.

\section{METHODOLOGIES:}

\subsection{GSM Based AMRS:}

Among the various implementations of Automated Meter Reading System the main modules that make difference are the Automated Meter Reading Module and the Communication Module. The following systems consider the GSM Network for Communication purposes. GSM network provides a global coverage across countries thus enabling communication to every nook and corner without the need to implement a new communication infrastructure solely for this purpose. Apart from seamless coverage the GSM technology also provides services like SMS (Short Message Service) and GPRS (General Packet Radio Service) for requesting and retrieving reading from individual houses back to the energy provider wirelessly. Moreover the GSM network is a more efficient, reliable and secure communication standard that is being widely used for more than several years now without any technical issues. The low cost, simple setup, wide operating distance, less human intervention are some of the other salient features of this GSM based system. One of the implementations of this system has a GSM modem and a P2C 
(Power to Communication) interface card installed inside it and is interfaced externally through RS232 from which the meter readings are obtained. This method suggests the tampering of the existing energy meter and integrating a P2C interface card to it, however the tampering of the energy meter is against the government law in most countries and could be implemented widely if and only if the existing meters where replaced by the government. The SMS system is used for requesting and retrieving energy meter readings from every house at anytime and even for cutting off the electricity connection if the bill remains unpaid. The user is also intimated, given feedback and able to check the status of his meter from anywhere in the world by using the SMS system ${ }^{[1][2]}$. Another implementation of the AMRS as shown in Fig. 1 is very much similar to our previous system but for it includes a RTC (Real Time Clock) for round the clock time stamping even on power failure (powered by a $3 \mathrm{~V}$ CMOS Battery) and EEPROM to store readings history. It also has a PC with GSM Receiver at the energy provider end that acts as the billing point. AT commands set which stands for attention terminal are used by energy meter to communicate with the GSM Modem. Both of these systems provide E-Commerce features like billing, printing, etc and an interface to view and analyze the history of all billings ${ }^{[3][4]}$.

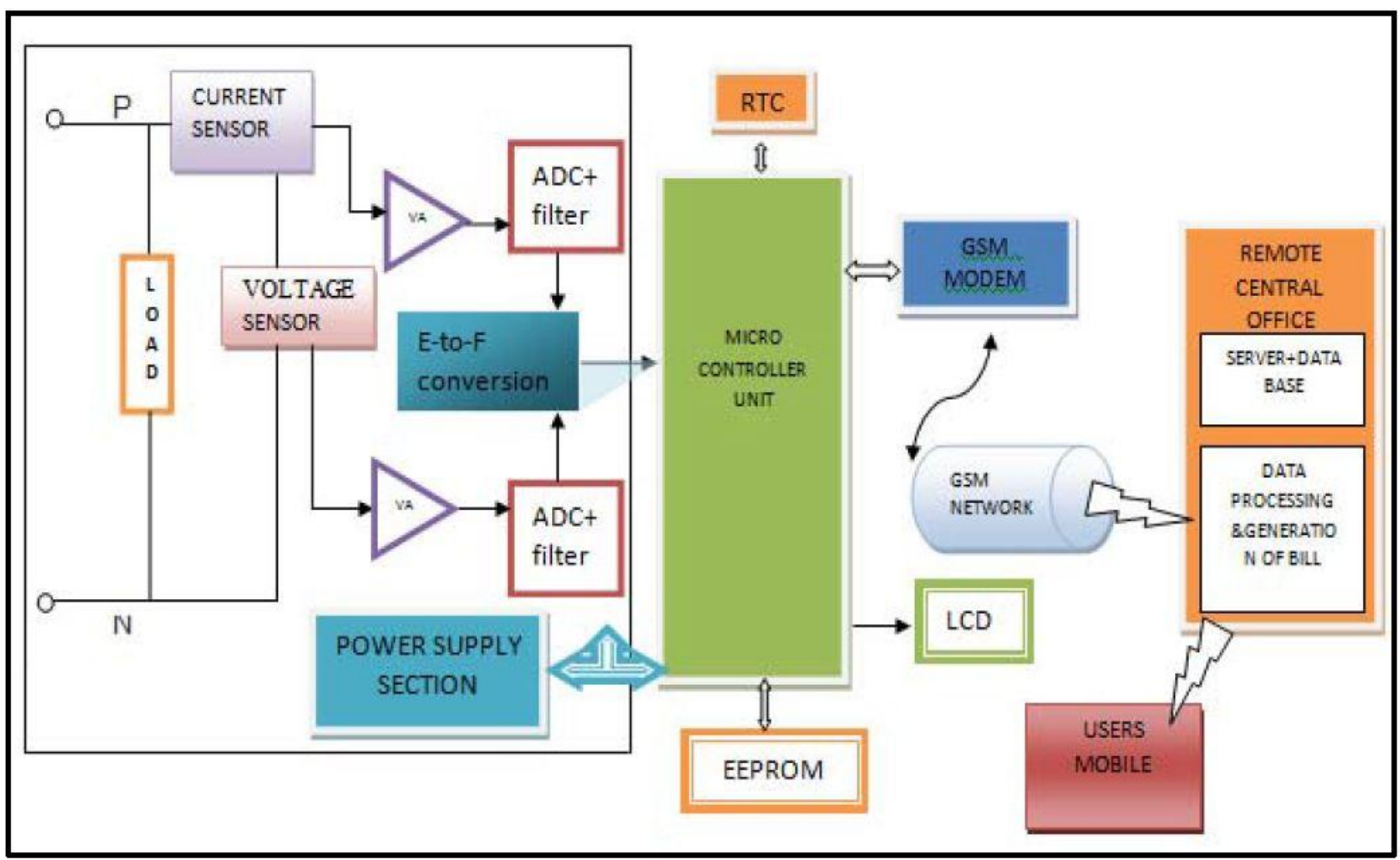

Fig. 1 Detailed Design Blocks. ${ }^{[2]}$

\subsection{Zigbee Based AMRS:}

ZigBee is a collection of communication protocols used to build small personal networks using low power digital radios. and is based on IEEE 802.15.4 standard. The range of an average ZigBee based device is restricted to 10 - 100 meters and can be further extended using a mesh network of ZigBee devices. The low cost allows the technology to be widely deployed in wireless control and monitoring applications. ZigBee was designed to provide high data throughput in applications where the duty cycle is low and low power consumption is an important consideration. The rate of ZigBee is definite with $250 \mathrm{kbit} / \mathrm{s}$, best suited for intermittent data transmissions from a sensor or input device ${ }^{[14]}$. The structure of WAMRS using ZigBee is shown in Figure 2.

ZigBee digital power meter is installed in every consumer unit and an electricity e-billing system at the energy provider side. The ZigBee digital power meter is a single phase digital $\mathrm{kWh}$ power meter with embedded ZigBee modem which sends the power usage reading using information back to the energy provider wirelessly. At the power provider side an e-billing system is used to manage all received meter reading, compute the billing cost, update the data base and to be published billing notification to its respective consumer through wireless.

Another way of using ZigBee in automated meter reading system is a system that consists of control terminal, GPRS module and user metering module. ZigBee network is for short distance communication, and GPRS is networks for remote communication. Clustering structure is used to reduce the redundancy in data transmission. The software is designed with the aim of saving energy with every communication protocol. ZigBee devices are cheap, easy to operate and consumes less energy but they are extremely limited in resources including processing, memory, and power. They also have a short operating range ${ }^{[5]}$. 


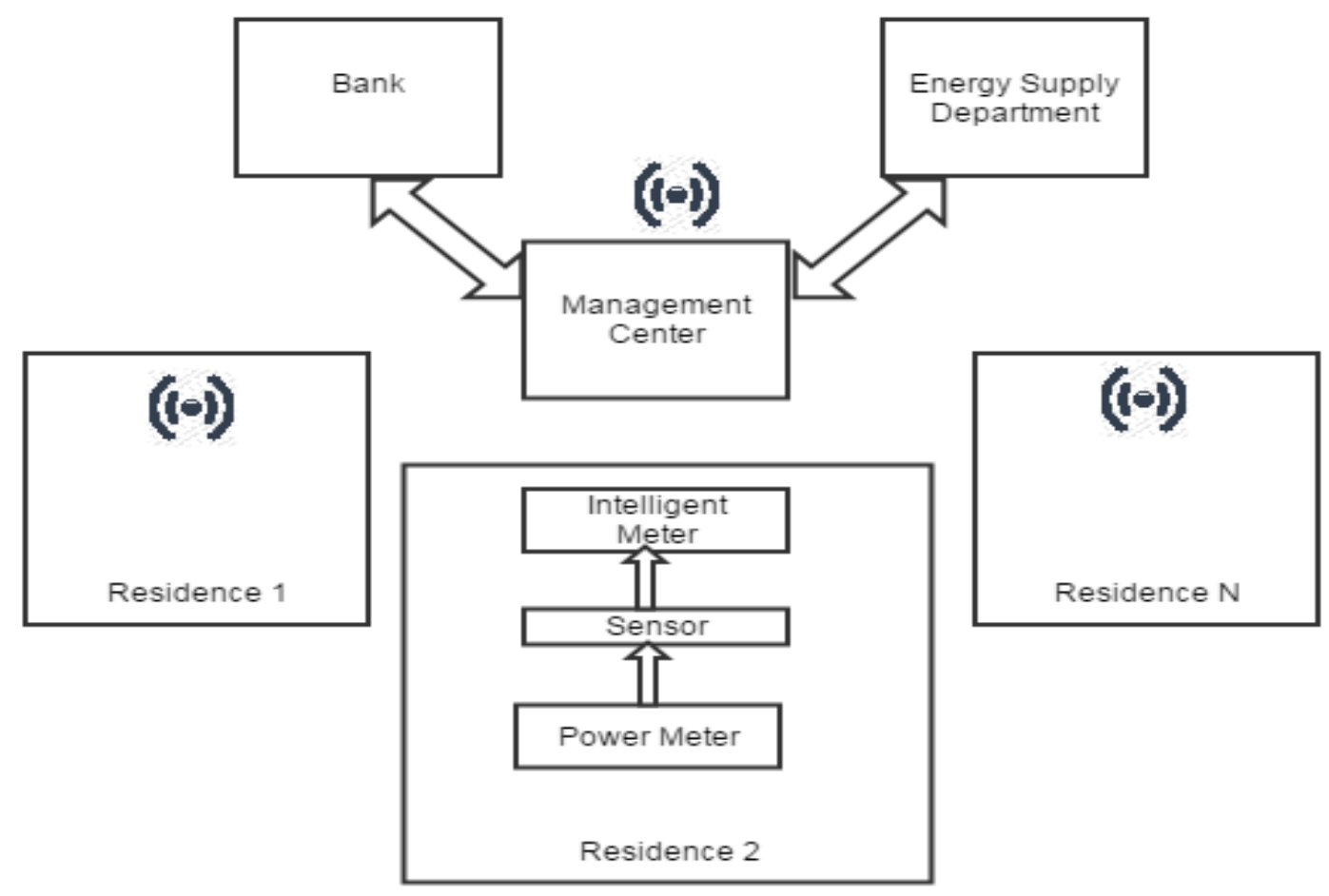

Fig. 2 Structure diagram of WAMRS. ${ }^{[5]}$

\subsection{AMRS Using SCADA System:}

SCADA (Supervisory Control and Data Acquisition) is a system operating with coded signals over communication channels so as to provide control of remote equipment. It is a category of software application program for process control, the gathering of data in real time from remote locations in order to control equipments.

SCADA refers to the combination of telemetry and data acquisition. SCADA includes the collecting of the information via a RTU (remote terminal unit), PLCs (programmable logic controllers) and IEDs (intelligent electronic devices), transferring it back to the central site, carrying out any necessary analysis and control and then displaying that information on a number of operator screens or displays. The D-SCADA control algorithm and distribution security monitoring is demonstrated in Fig 3. A system using SCADA isolates and precisely locates faults and optimise for energy saving. Centralised monitoring and control to manage multiple facilities from a single location and remote software administration for better control is possible. Systems using SCADA are prone to network attacks ${ }^{[6]}$.

\subsection{AMRS using Power Line Communication:}

Power Line Communication Systems use the existing power cables as a communication medium. This ultimately allows us to both control the devices remotely and also retrieve data from it in a half duplex manner ${ }^{[15]}$. PLC is like any other communication technology whereby a sender modulates the data to be sent, injects it onto medium, and the receiver demodulates the data to read it. The main advantage of PLC is that the existing wiring can be re-used. Thus Power Line Communication can be used to transfer meter readings data to the central servers along with the use of GSM/GPRS. The data from every meter is collected using PLC. This data is then transferred to a central server using GPRS connectivity. The server in turn feeds the data to service provider's billing servers thus creating a totally automated and reliable measurement and billing infrastructure. Value added services can be used to send alerts for prepaid users, SMS facility to switch off gas supply, alerts on gas leak detection and automatic switching off gas supply, credit card payment facility.

The communication between concentrator and collectors is done using Power Line Carrier(PLC) as shown in Fig 4. A collector is used to store and process the data from different electric meters with the supervision of the concentrator. A concentrator sends commands to collector to receive meter readings periodically. 


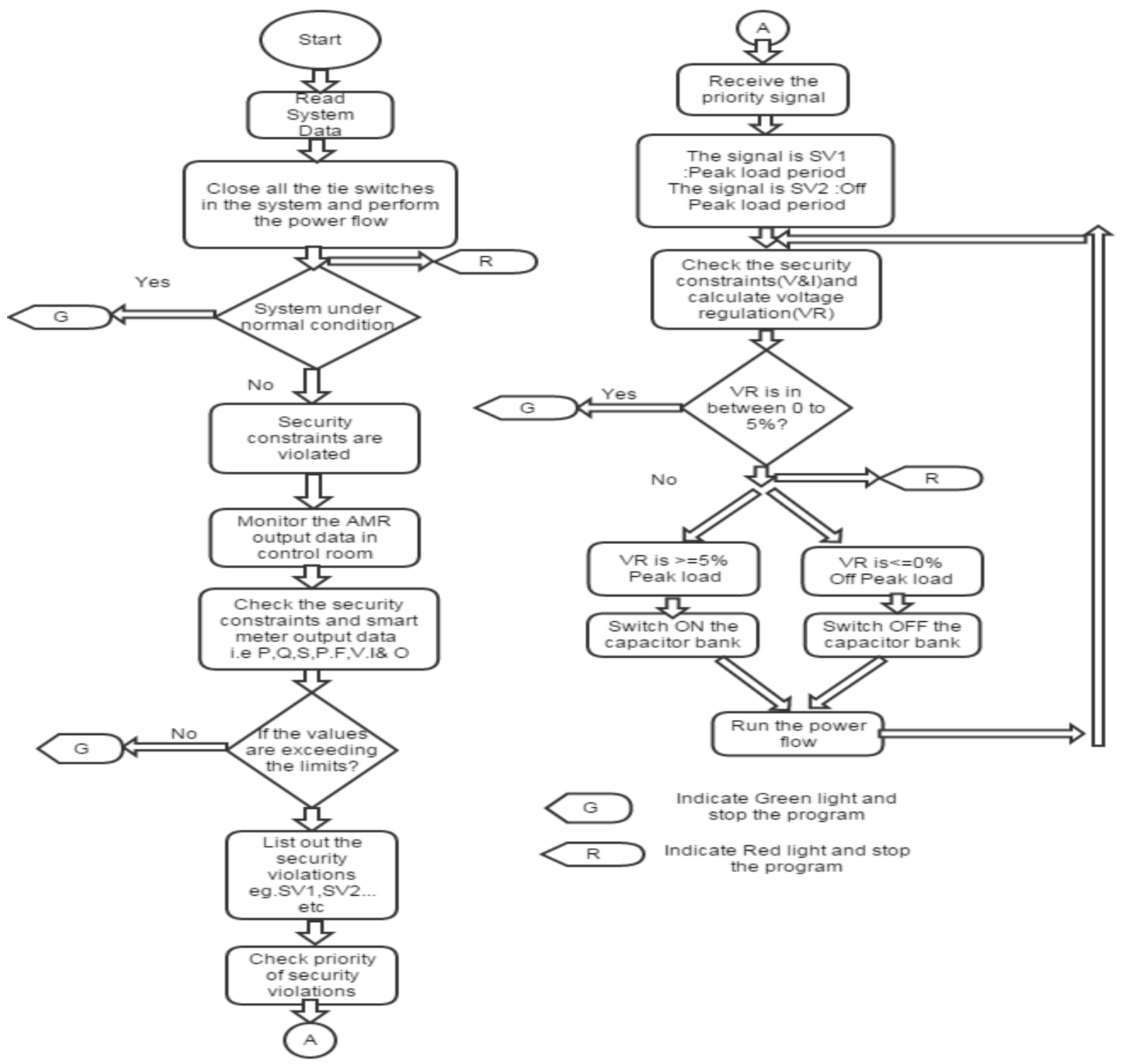

Fig. 3 AMR based distribution security monitoring and D-SCADA control algorithm. ${ }^{[6]}$

The two major factors causing PLC meter readings unstable are the electric network signal attenuation and random interference. Because of the attenuation, wave carrier reading system cannot read meters reliably if there isn't any relay or anti-attenuation techniques. The existence of random interference equals to increasing the attenuation of the electric network and decreasing the receiving sensitivity, if the sensitivity of the meter reading device is constant. This affects the meter reading system severely. A system using PLC can make use of existing electrical wires and has a better communication range than wireless communication. PLC is easy to install and maintain. It cannot consistently read the data. The long term interference on the electric network causes the household appliances to over-heat, motor output decreasing etc ${ }^{[7]}$.

\subsection{AMRS using WiMAX Technology:}

WiMAX (Worldwide Interoperability for Microwave Access) is a wireless communications standard designed to provide 30 to 40 megabit-per-second data rates. WiMAX supports mobile, nomadic and fixed wireless applications. WiMAX brings with it the ability to transmit over far greater distances and to handle much more data.

The reading unit identifies the disk rotation of the energy meter and the data is stored in a microcontroller. So it is not required to change the current analog energy meter. An external module is added with the current energy meter. In the communication unit WiMAX transceiver is used for wireless communication between meter end and the server end because of its wide coverage area. In the data receiving and processing unit meter reading is collected from the transceiver which is controlled by another microcontroller. There is a computer application that will take the data from the microcontroller. This will also help to avoid any tampering or break down of energy meter. The conceptual diagram of the proposed system 


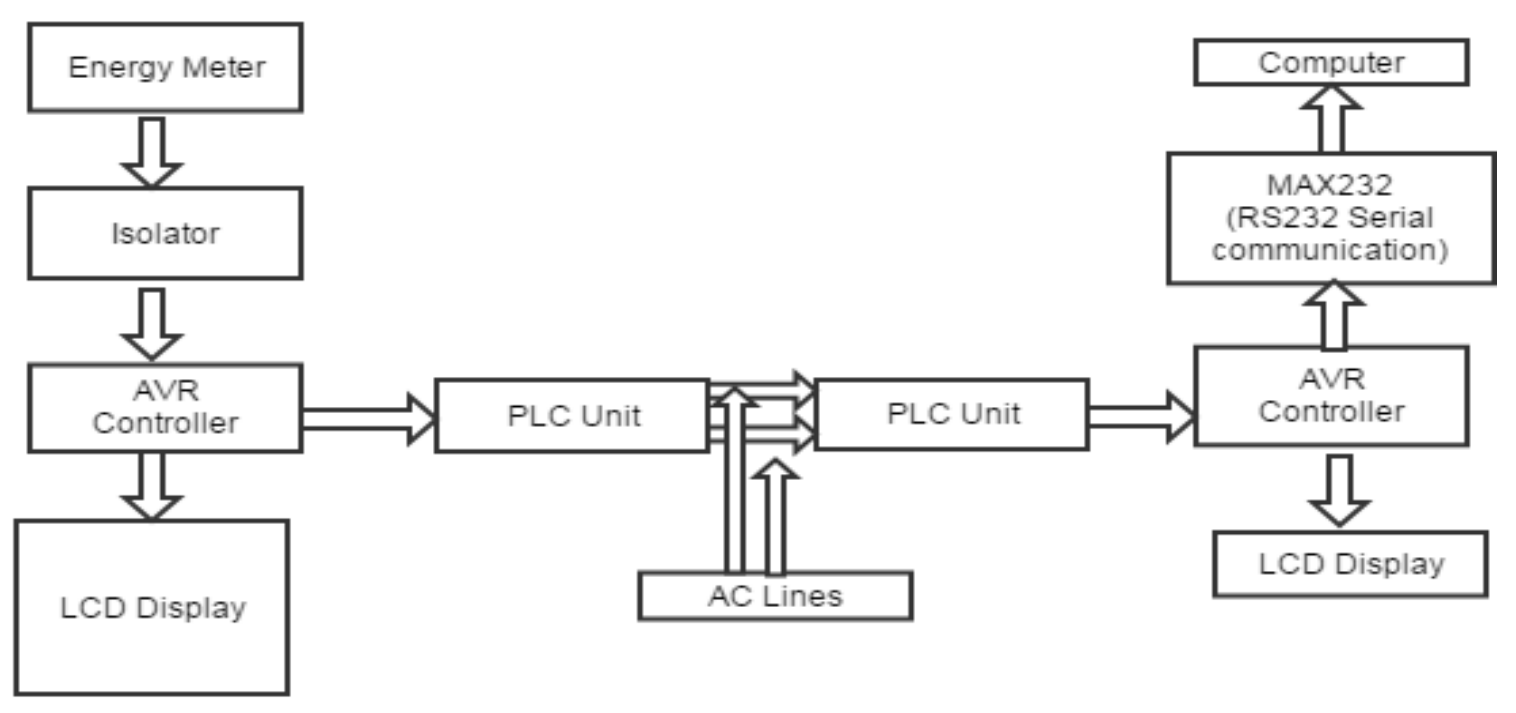

Fig 4.Block Diagram of AMRS Using Power Line Communication. ${ }^{[7]}$

in shown in Fig 5.This system is cheap, flexible and supports high speed data transfer over long distances. WiMAX has security vulnerabilities in both PHY and MAC layers, exposing to various classes of wireless attack including interception, fabrication, modification, and replay attacks. Weather conditions like rain could interrupt the signal. Other wireless equipment could cause interference ${ }^{[8]}$.

\subsection{Mixed Type}

The following system automates the meter reading process by capturing images of the Energy meter and transferring them to Customers using ZigBee technology. It generates the bill and sends the current reading and previous meter reading as a SMS to the customer. Matlab is used to process the image and get the digits out.C328R serial VGA Camera is placed in front of the energy meter to capture images which is transferred to the server where the characters and numbers are recognized. This also avoids tampering of energy meter. For extraction of digits from the whole image only the digits plate is cropped. It undergoes preprocessing such as RGB to grey, B/W image \& then each digits are segmented. After this, each digit undergoes the process of grid technique to find the value of this digit with previously stored digits in database \& numbers are recognized easily. This reading gives the current meter reading. Use of Matlab renders the system costly. The system consumes less power but has a short range and low data speed. ${ }^{[9]}$

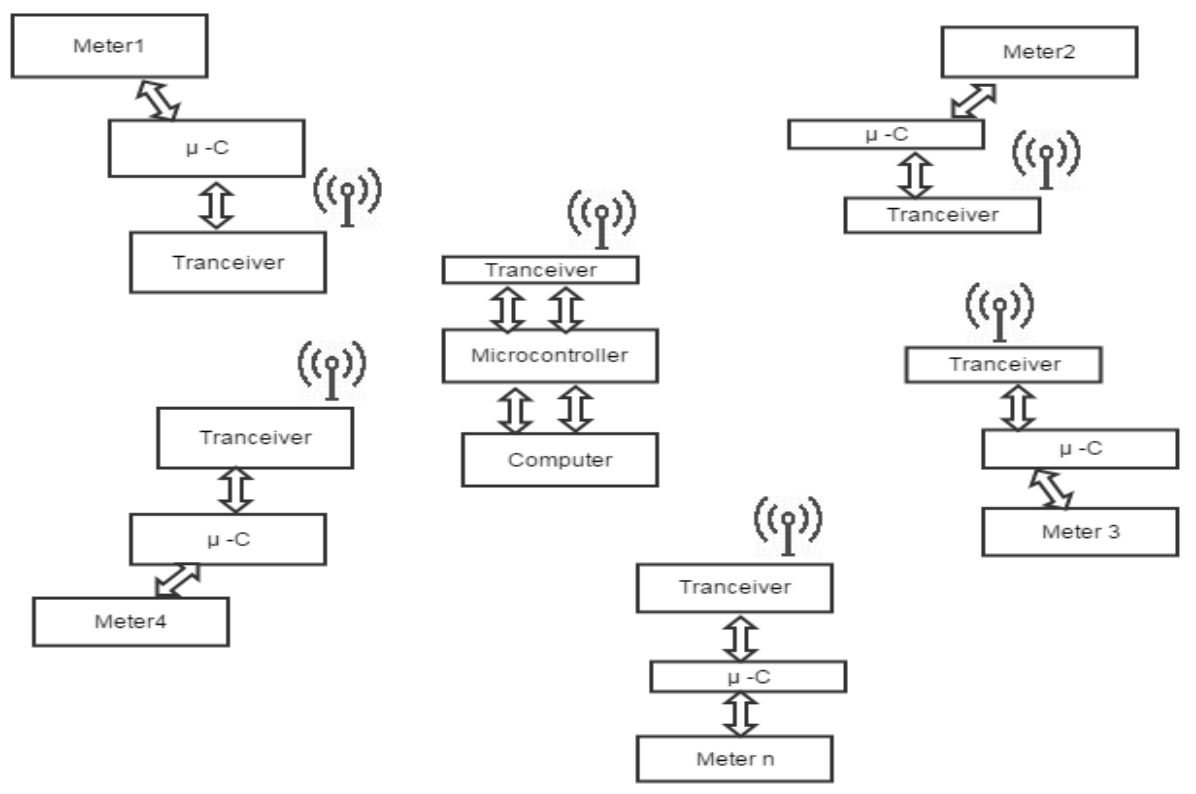

Fig. 5 Conceptual Diagram of proposed AMR. ${ }^{[8]}$ 
The following provides two variants of the automatic meter reading system using ZigBee and GSM: 1.PIC Microcontroller to count the pulses generated by the metering IC.2.Wireless Sensor Networks(WSN) to detect, process and store sensor output.

In the first system, the metering IC creates the output in the form of pulses which are counted using the default timer of PIC microcontroller unit. A TTL inverter circuit is used to reverse the produced pulse before applying to the counter. The PIC microcontroller is programmed using software interfacing for reading the data from the metering IC. The technology used to send data to the Base station is ZigBee which in turn uses GSM modem to communicate with Main EB Station. ${ }^{[10]}$

In the second system, the sensor network consists of multiple detection stations called sensor nodes (contains transducer, microcomputer, transceiver), each of which is small, lightweight and portable. The transducer generates electrical signals based on sensed physical effects and phenomena. The microcomputer processes and stores the sensor output. The transceiver receives commands from a central computer and transmits data to that computer. Finally all the data is sent to the main station that broadcast messages of bills of individual houses, due dates for payments, confirmation of bill payments.

WSN eliminate a lot of wiring which is not in the case of PIC microcontroller which requires a lot of wires. Wireless Sensor Networks are very expensive compared to PIC's. WSN is not secure. On the other hand, there is no major security concern in PIC.PIC have more complex architecture compared to WSN. ${ }^{[11]}$

The automation of meter reading is achieved using GSM and ARM Controller. The system uses ARM processor to process the input data, compute the bill and UART for data exchange with GSM modem. It consists of energy measuring unit for tamper detection, relay control unit for remote switching of the system, wireless communication module for data transmission and utility control center at the server side to collect data, process and send SMS to customer. The system reads the input pulses and after counting specific number of pulses it increments the internal counter by one which indicates the number of units consumed. ARM controller sends the no of units to the UART, which is then sent to the GSM Modem. The GSM modem sends this data to the service provider using SMS. User's supply can be disconnected remotely in case of unpaid bills. Data collection and manipulation task is made fast and easier. ${ }^{[12]}$

The final system under mixed type consists of a remote control terminal, a GPRS module and a user metering module. .User meter reading module consists of three parts namely intelligent instrument data acquisition module, data storage and data transfer module. The data collected is buffered and sent to the remote terminal through wireless network. The network structure of remote AMRS in shown in Fig 6.The function of communication of GPRS module in short distance uses the CC2430 chip and remote communications uses SIM300 chip. To reduce the redundancy in data transmission, clustering structure is used. ZigBee network is used for short distance communication, and GPRS is used for remote communication. They can combine with each other, through the network's gateway to achieve a user meter copying to control and transfer in distance. This software is designed with the aim of saving energy with every communication protocol. This system is inexpensive, works with water, electricity and gas meters and is greatly secure. The capacity of the buffer area of data, affect the quality of sending and receiving. It does not support remote termination of the supply ${ }^{[14]}$.

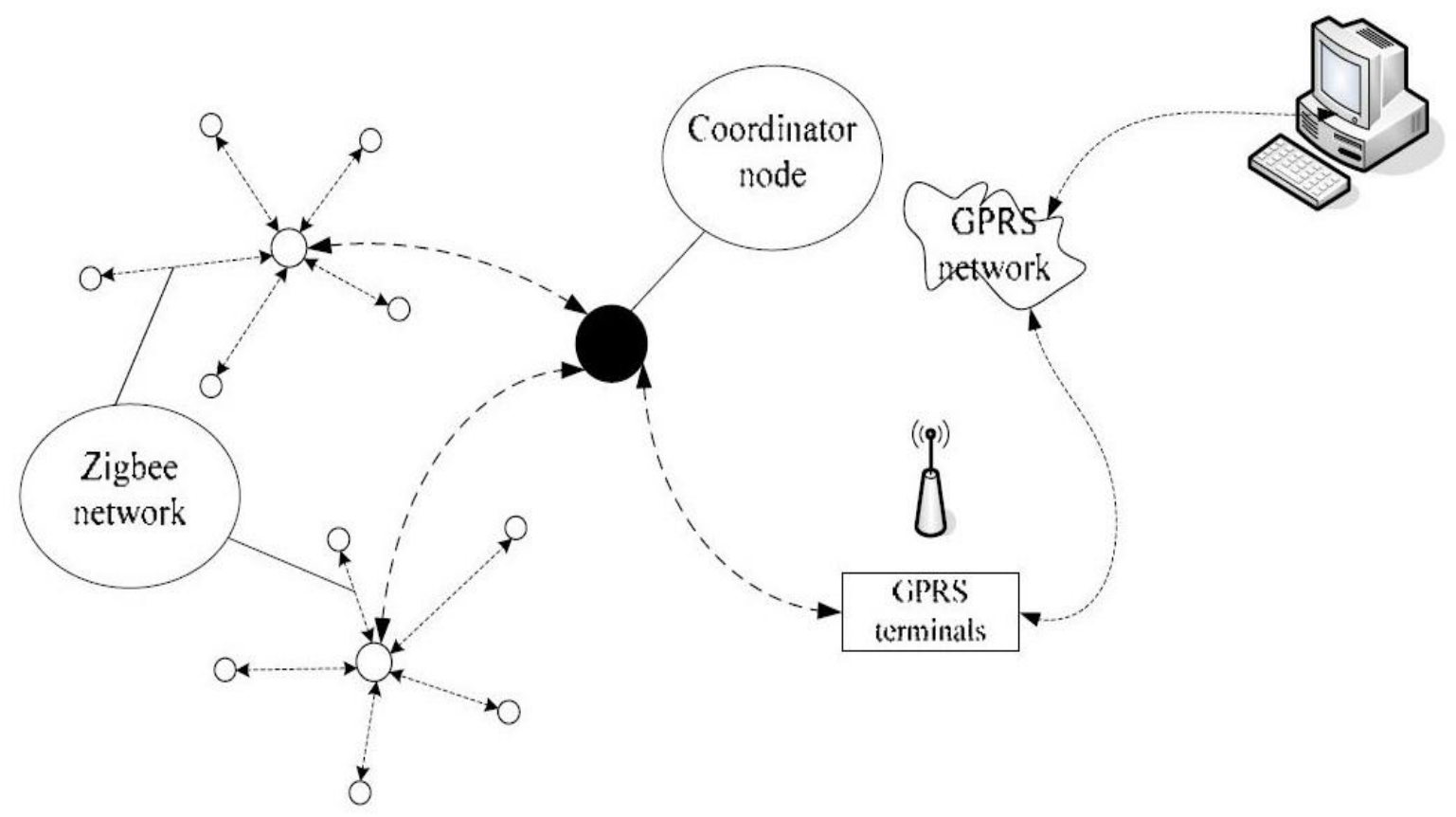

Fig. 6 Network Structure. ${ }^{[14]}$ 


\section{COMPARISON}

Table 1. Consolidated Comparison of all the Systems

\begin{tabular}{|c|c|c|c|c|c|}
\hline $\begin{array}{c}\text { Technology } \\
\text { Used }\end{array}$ & Cost & Feasibility & Reliability & Coverage & $\begin{array}{c}\text { Communication } \\
\text { Protocol }\end{array}$ \\
\hline GSM & Low & Most Feasible & High & High & Stable \\
\hline ZigBee & Medium & Small Scale & Low & Low & Least Stable \\
\hline SCADA & High & Not Feasible & High & Low & Stable \\
\hline PLC & Low & Least Feasible & Low & Very High & Very Stable \\
\hline WiMAX & Medium & Small Scale & Medium & Low & Stable \\
\hline Mixed & Varies & $\begin{array}{c}\text { Feasible if GSM } \\
\text { is a part of it }\end{array}$ & Varies & $\begin{array}{c}\text { High if GSM } \\
\text { is a part of it }\end{array}$ & Varies \\
\hline
\end{tabular}

\section{FUTURE SCOPE}

Though many have tried implementing a country wide AMRS, it is still an unreachable goal, many factors like cost, feasibility and mainly the need to replace the existing system have hindered its development. At least in near future, the cost involved in the building of this system could be minimized by using more efficient technology and commercializing it by production in a large scale thus reducing the production costs tremendously and hence making it more feasible to be implemented worldwide. The system could be made more Smart by allowing the user to check for the power consumption and alert him on his power consumption and also allows him to turn off the various devices from his mobile application itself hence conserving energy and saving money. Moreover if real time data feeds of the energy usage were tracked and stored in a centralized data centre it will enable the government or the energy provider to predict the energy demand trends consequently ensuring uninterrupted supply of electricity and reducing the unexpected load on the electricity grid making it more tolerant to failures.

\section{CONCLUSION}

Based on all the systems surveyed, their advantages and drawbacks, this paper presents the features that make up an ideal AMR system and provides a overall insight of the various methodologies applied for AMR so far thus providing a base for further research in this area. Based on the performance criteria considered, several systems where compared and now we come to a conclusion that, when it comes to Reliability and Feasibility GSM topped the charts but when Coverage and Communication protocol used comes in to picture PLC steals the stage. Other suggestion would be to include a web/mobile interface that gives a real-time feedback of the energy consumption, maybe even give the amount of energy consumed by every device in every room and also give energy conservation tips too, based on the data obtained. The real-time data thus obtained can also be used to build a prediction model over it, for predicting the energy demands trends of the future, over any given time period.

\section{REFERENCES}

[1] H.G.Rodney Tan,IEEE, C.H.Lee and V.H.Mok, IEEE , "Automatic Power Meter Reading System Using GSM Network", The 8th International Power Engineering Conference,pp.465-469,IPEC 2007.
[2] Ashna. K,Sudhish N George, "GSM Based Automatic Energy Meter Reading System with Instant Billing", 9781-4673-5090-7/13@2013 IEEE, pp 65-71.

[3] H. M. Zahid Iqbal ,M.Waseem,Dr. Tahir Mahmood, "Automatic Energy Meter Reading using Smart Energy Meter".

[4] Abhinandan Jain,Dilip Kumar,Jyoti Kedia, "Design and Development of GSM based Energy Meter", International Journal of Computer Applications (0975 888), Volume 47- No.12, pp. 41-45,June 2012.

[5] S.Arun,Dr.Sidappa Naidu, "Design and Implementation of Automatic Meter Reading System Using GSM,ZIGBEE through GPRS”, ISSN: 2277 128X ,International Journal of Advanced Research in Computer Science and Software Engineering, Volume 2, Issue 5, pp. 321-325,May 2012.

[6] T. Ananthapadmanabha, A. D. Kulkarni, Madhvaraja, and A. P. Suma, "Automatic meter reading (AMR) based distribution security monitoring and distributionsupervisory control and data acquisition (D-SCADA) control", Journal of Electrical and Electronics Engineering Research Vol. 3(6), pp. 108-120, August 2011.

[7] Poonam Borle, Ankita Saswadkar, Deepali Hiwarkar, Rupali S. Kad, ”Automatic Meter Reading For Electricity Using Power Line Communication, International Journal of Advanced Research in Electrical, Electronics and Instrumentation Engineering, Vol. 2, Issue 3,pp. 982987,March 2013.

[8] Tanvir Ahmed, MD Suzan Miah, MD. Manirul Islam, MD. Rakib Uddin," Automatic Electric Meter Reading System: A Cost Feasible Alternative Approach In Meter Reading For Bangladesh Perspective Using Low-Cost Digital Watt Meter And WIMAX Technology".

[9] Manisha V Shinde, Pradip W Kulkarni, "New Development in Energy Meter Reading System", ISSN: 2349-3371,International Journal of Scientific Research and Management Studies (IJSRMS),Volume 1 Issue 4, pp. 124-129.

[10] S.Arun,Dr.Sidappa Naidu, "Hybrid Automatic Meter Reading System", ISSN: 2277 128X, International Journal of Advanced Research in Computer Science and 
Software Engineering, Volume 2, Issue 7, pp.361-365, July 2012.

[11] Amrutha Kotasthane,Hate S.G, "Implementation of Automatic Meter Reading System Using Wireless Sensor Network", ISSN: 2278-1323,International Journal of Advanced Research in Computer Engineering \& Technology (IJARCET), Volume 2, Issue 12, pp. 30303032,December 2013

[12] Bharat Kulkarni,"GSM Based Automatic Meter Reading System Using ARM Controller",International Journal of Emerging Technology and Advanced Engineering, ISSN 2250-2459, Volume 2, Issue 5, pp.446-448,May 2012.
[13] Li Quan-Xi, Li Gang, ”Design of remote automatic meter reading system based on ZigBee and GPRS", ISBN 978952-5726-10-7,Proceedings of the Third International Symposium on Computer Science and Computational Technology(ISCSCT '10), Jiaozuo,P.R.C.

[14] ZigBee Introduction, Wikipedia article on ZigBee http://en.wikipedia.org/wiki/ZigBee.

[15] PLC (Power Line Communication) Introduction, www.eetimes.com/document.asp?doc_id $=1279014$ 